\section{OPEN ACCESS}

Edited by:

Fernando Testai,

University of Illinois at Chicago,

United States

Reviewed by:

Joanna Maria Zajkowska,

Medical University of Bialystok, Poland

Alejandro Vargas,

Rush University Medical Center,

United States

*Correspondence:

Kiran T. Thaku

ktt2115@cumc.columbia.edu

Specialty section:

This article was submitted to

Neurocritical and Neurohospitalist

Care,

a section of the journal

Frontiers in Neurology

Received: 07 May 2020

Accepted: 29 June 2020

Published: 10 July 2020

Citation:

Radmard S, Epstein SE, Roeder HJ,

Michalak AJ, Shapiro SD, Boehme A,

Wilson TJ, Duran JC, Bain JM,

Willey JZ and Thakur KT (2020)

Inpatient Neurology Consultations

During the Onset of the SARS-CoV-2

New York City Pandemic: A Single

Center Case Series.

Front. Neurol. 11:805

doi: 10.3389/fneur.2020.00805

\title{
Inpatient Neurology Consultations During the Onset of the SARS-CoV-2 New York City Pandemic: A Single Center Case Series
}

\section{Sara Radmard ${ }^{1}$, Samantha E. Epstein ${ }^{1}$, Hannah J. Roeder ${ }^{1}$, Andrew J. Michalak ${ }^{1}$, Steven D. Shapiro ${ }^{1}$, Amelia Boehme ${ }^{2}$, Tommy J. Wilson ${ }^{1}$, Juan C. Duran ${ }^{3}$, Jennifer M. Bain ${ }^{3}$, Joshua Z. Willey ${ }^{1}$ and Kiran T. Thakur ${ }^{1 *}$}

'Department of Neurology, New York-Presbyterian/Columbia University Irving Medical Center, New York, NY, United States, 2 Department of Neurology and Epidemiology, Sergievsky Center, Columbia University Irving Medical Center, New York, NY, United States, ${ }^{3}$ Department of Neurology, Division of Child Neurology, New York-Presbyterian/Columbia University Medical Center, New York, NY, United States

Objective: Severe acute respiratory syndrome coronavirus 2 (SARS-CoV-2) primarily causes respiratory illness. However, neurological sequelae from novel coronavirus disease 2019 (COVID-19) can occur. Patients with neurological conditions may be at higher risk of developing worsening of their underlying problem. Here we document our initial experiences as neurologic consultants at a single center quaternary hospital at the epicenter of the COVID-19 pandemic.

Methods: This was a retrospective case series of adult patients diagnosed with SARS-CoV-2 who required neurological evaluation in the form of a consultation or primary neurological care from March 13, 2020 to April 1, 2020.

Results: Thirty-three patients (ages 17-88 years) with COVID-19 infection who required neurological or admission to a primary neurology team were included in this study. The encountered neurological problems associated with SARS-CoV-2 infection were encephalopathy (12 patients, 36.4\%), seizure (9 patients, $27.2 \%)$, stroke (5 patients, $15.2 \%)$, recrudescence of prior neurological disease symptoms (4 patients, $12.1 \%$ ), and neuromuscular (3 patients, 9.1\%). The majority of patients who required evaluation by neurology had elevated inflammatory markers. Twenty-one (63.6\%) patients were discharged from the hospital and 12 (36.4\%) died from COVID-19 related complications.

Conclusion: This small case series of our initial encounters with COVID-19 infection describes a range of neurological complications which are similar to presentations seen with other critical illnesses. COVID-19 infection did not change the overall management of neurological problems.

Keywords: neurology, novel coronavirus, COVID-19, severe acute respiratory syndrome coronavirus 2, inpatient consults 


\section{INTRODUCTION}

Novel coronavirus disease 2019 (COVID-19) is a global pandemic caused by the pathogen severe acute respiratory syndrome coronavirus 2 (SARS-CoV-2) (1). SARS-CoV-2 causes a respiratory illness that primarily manifests in symptomatic individuals as fever, cough, and myalgias. More severe infection leads to pneumonia, acute respiratory distress syndrome (ARDS) or a large systemic inflammatory cytokine response (2), resulting in multi-organ damage.

The full spectrum of neurological manifestations of COVID19 not fully elucidated. Other coronavirus strains are associated with seizures, encephalitis, and demyelinating syndromes (3). Proposed mechanisms of disease for these strains include entry into the central nervous system through breakdown of the blood brain barrier and invasion through peripheral nerves (3). For SARS-CoV-2 in particular, limited knowledge exists regarding its effect on the nervous system and impact on pre-existing neurological disorders. Case series have primarily reported symptoms of hyposmia, hypogeusia, dizziness, encephalopathy, and delirium $(4,5)$. Only one case of new-onset seizure has been reported (4). A few studies have noted an increase incidence in stroke with COVID-19 infection (4, 6-8). including in young adults (9).

With the rapid spread of SARS-CoV-2, neurologists will increasingly be consulted to guide care for patients with existing neurological conditions and new neurological symptoms due to COVID-19. We conducted a single-center retrospective review of our first experiences with COVID-19 infection in inpatients with or without pre-existing neurological disorders. We document 33 patients who had neurological symptoms while hospitalized with COVID-19 infection and summarize the associated laboratory and ancillary data performed. We discuss unique cases that raise questions about the nuances of management in a neurological population.

\section{METHODS}

\section{Patients}

Adult inpatients diagnosed with COVID-19 infection by nasopharyngeal swab reverse transcription polymerase chain reaction (RT-PCR) and required neurological evaluation by consultation or admission for primary neurological care were included in this single-center retrospective case-series study at Columbia University Irving Medical Center (CUIMC)-New York Presbyterian Hospital. Cases from March 13, 2020 to April 1, 2020. Clinical information was gathered by patient chart review, including demographics, medical history, clinical presentation, clinical course, radiographic imaging, laboratory values, hospital duration, therapeutic agents, and outcome. Pulmonary disease includes pre-existing obstructive lung disease, restrictive lung disease, and/or chronic tobacco use. Cardiovascular disease includes hyperlipidemia or hypercholesterolemia, hypertension, coronary artery disease (CAD), peripheral arterial disease, diabetes mellitus, and morbid obesity. Renal disease is defined as pre-existing chronic kidney disease or end-stage renal disease requiring dialysis. Immune suppression is defined as autoimmune disorder on immunosuppressive therapy, immunodeficiency from HIV/AIDS, or history of cancer on chemotherapy. Encephalopathy is defined as depressed consciousness, fluctuations in consciousness, or impaired consciousness leading to language comprehension deficits excluding aphasia.

\section{Standard Protocol Approvals and Patient Consents}

This study was approved by the Institutional Review Board (IRB) of CUIMC. Informed consent was obtained for the two cases delineated in detail. Informed consent was not required by our institutional IRB for the remainder of the participants.

\section{Statistical Analysis}

Statistical associations were calculated using mean, median, range, and standard deviation. All statistical analyses were carried out using SAS (version 9.4, trademarks of SAS Institute Inc., Cary, NC, USA).

\section{RESULTS}

\section{Patients}

Thirty-three patients were included in this study of which 1 was near adult age (17 years). The mean age of patients was $56.1( \pm 17.1)$ years (Table 1). Twenty-two patients $(66.7 \%)$ had a known neurological condition, including epilepsy $(n=6)$, ischemic stroke $(n=5)$, dementia $(n=5)$, developmental delay $(n=4)$, myasthenia gravis $(n=2)$, and multiple sclerosis or other central autoimmune disease $(n=2)$. Seventeen (51.5\%) had cardiovascular comorbidities, 5 (15.2\%) pulmonary disease, and 7 (21.2\%) renal disease (Table 1). Ten (30.3\%) were immunocompromised due to transplant $(n=2)$, cancer with or without chemotherapy $(n=7)$, and autoimmune disorder on immunosuppression $(n=1)$ (Table 1). These cases represented $3.9 \%$ of inpatients who had tested positive for SARS-CoV-2 during the 2-week time course.

\section{Clinical Presentation}

The most frequent reason for neurology consultation was encephalopathy $(n=12,36.4 \%)$. Encephalopathy was attributed to toxic/metabolic derangements, systemic infection, or concern for cytokine storm. Continuous EEG (cEEG) was obtained for $7(21.2 \%)$ patients to rule out seizure as cause of the encephalopathy. Continuous EEG monitoring showed triphasic waveforms in one patient with hepatic encephalopathy (case 18 ) and one patient with acute renal failure due to septic shock (case 16).

The second most frequent reason for neurology consultation was seizure $(n=9,27.2 \%)$, of which $6(66.7 \%)$ patients had first seizure of life (Table 2). Three patients with first seizure of life had cEEG monitoring which showed frontotemporal seizures, parasagittal seizures, and marked attenuation (cases 12, 13, and 21 , respectively). Only one patient with first seizure of life had a history of neurological disease, which was possible anoxic brain injury from ventricular fibrillation arrest (case 21). Five (83.3\%) patients with first seizure of life had inflammatory marker data. 
TABLE 1 | Patient demographics, medical history, neurological clinical presentation, COVID-19 laboratory markers, therapeutic interventions, and outcomes.

\begin{tabular}{|c|c|}
\hline \multicolumn{2}{|c|}{ Patient demographics \& clinical presentation } \\
\hline Age (y), mean (SD), median, range & $56.1(17.1), 60,17-88$ \\
\hline \multicolumn{2}{|l|}{ Sex } \\
\hline Female & $13(39.4 \%)$ \\
\hline Male & $20(60.6 \%)$ \\
\hline \multicolumn{2}{|l|}{ Ethnicity, $n$ (\%) } \\
\hline White & $10(30.3 \%)$ \\
\hline Hispanic & $14(42.4 \%)$ \\
\hline African American & $6(18.2 \%)$ \\
\hline Unknown & $3(9.1 \%)$ \\
\hline \multicolumn{2}{|l|}{ Neurological symptom, $n$ (\%) } \\
\hline Encephalopathy & $12(36.4 \%)$ \\
\hline Seizure & $9(27.2 \%)$ \\
\hline Stroke & $5(15.2 \%)$ \\
\hline Recrudescence & $4(12.1 \%)$ \\
\hline Neuromuscular & $3(9.1 \%)$ \\
\hline \multicolumn{2}{|l|}{ Medical History, $n$ (\%) } \\
\hline Pulmonary disease & $5(15.2 \%)$ \\
\hline Cardiovascular disease & $17(51.5 \%)$ \\
\hline Renal disease & $7(21.2 \%)$ \\
\hline Immune Suppression & $10(30.3 \%)$ \\
\hline Intubation required & $8(24.2 \%)$ \\
\hline \multicolumn{2}{|l|}{ Laboratory values, mean (SD) } \\
\hline Peak ferritin & $1438.8(1685.0)$ \\
\hline Peak IL-6 & $82.2(111.9)$ \\
\hline Peak D-dimer & $6.2(6.9)$ \\
\hline Peak procalcitonin & $15.7(63.6)$ \\
\hline Peak C-RP & 146.7 (99.8) \\
\hline \multicolumn{2}{|l|}{ Therapeutic interventions, mean (\%) } \\
\hline Hydroxychloroqine & 15 (45.5\%) \\
\hline Azithromycin & 11 (33.3\%) \\
\hline Remdesivir & $2(5.3 \%)$ \\
\hline Tocilizumab & $2(6.1 \%)$ \\
\hline \multicolumn{2}{|l|}{ Outcomes, mean (SD) } \\
\hline Length of hospital stay (d) & $16.6(16.8)$ \\
\hline Death & 12 (36.4\%) \\
\hline Discharged from hospital & $21(63.6 \%)$ \\
\hline Average age at death (y) & $61.4(10.6)$ \\
\hline
\end{tabular}

$y$, year; d, day; SD, standard deviation; $n$, number; WBC, white blood count; IL-6, interleukin-6; C-RP, C-reactive protein.

All had elevated C-RP >100 milligram per liter $(\mathrm{mg} / \mathrm{L})$, four $(80 \%)$ ferritin $>1,000$ nanogram per milliliter $(\mathrm{ng} / \mathrm{mL})$, and four (80\%) IL-6 >30 picogram per $\mathrm{mL}$ (pg/mL) (Figure 1). Two patients with focal seizures had no evidence of structural lesion on CT head. MRI brain with and without contrast was normal for the pediatric first seizure of life patient (case 1). All patients with first seizure of life were treated with levetiracetam except for the pediatric patient who was discharged home on no antiepileptic medications.

Five $(15.2 \%)$ patients presented with acute cerebrovascular insults due to ischemia ( $n=3$; ages 45,57 , and 67 years), intracerebral hemorrhage ( $\mathrm{ICH})$ /intraventricular hemorrhage $(\mathrm{IVH})$ /subarachnoid hemorrhage $(\mathrm{SAH})(n=1)$, and subdural hematoma $(\mathrm{SDH})(n=1)$ (Table 2). All patients with ischemic strokes had cardiovascular risk factors (cases 8, 15, and 22). One patient had sickle cell trait (case 8).

Recrudescence of a prior neurological condition occurred in 4 (12.1\%) patients. Recrudescence was diagnosed if a patient had a prior neurological deficit that worsened in the setting of infection but improved back to baseline after symptomatic treatment of underlying infection.

\section{Laboratory and Radiographic Data}

Of the inflammatory marker levels obtained, ferritin was elevated in $75 \%(n=21 / 28)$ of patients, IL-6 in 57\% $(n=12 / 21)$, d-dimer in $65 \%(n=15 / 23)$, and C-RP in $90 \%(n=26 / 29)$ (Figure 2$)$.

Brain imaging was obtained for $20(60 \%)$ patients, including 3 (7.6\%) patients who underwent MRI. Relevant findings included acute SDH, ICH/IVH/SAH, acute ischemic strokes $(n=2)$, and meningioma with vasogenic edema $(n=1)$. The remainder showed chronic changes or were unremarkable. Of note, one patient who had several months of hyposmia and facial numbness presented with worsening of the symptoms. MRI brain with and without contrast was unremarkable with no abnormal contrast enhancement (case 14).

In an attempt to reduce exposure and preserve personal protective equipment (PPE), CSF testing was only pursued if there was another indication. As a result, CSF was only obtained in one case highlighted below for autoimmune encephalitis.

\section{Patient Outcomes}

Twenty-one $(63.6 \%)$ patients were discharged from the hospital and 12 (36.4\%) died. One 70-year-old male known epilepsy and intellectual disability from childhood meningitis presented with breakthrough seizures and died due to presumed cytokine storm and multiorgan failure. One patient with relapsing-remitting multiple sclerosis on natalizumab who presented with worsening bilateral leg weakness died due to severe ARDS and shock.

\section{Case 29}

A 74-year-old woman with a history of remote breast and pancreatic cancers, recent varicella zoster virus (VZV) radiculitis, and possible antibody negative autoimmune encephalitis was transferred to our institution for worsening mental status manifested as stupor and global aphasia. CSF studies were notable for a white blood cell count 12 per microliter $(\mu \mathrm{L})$ (99\% lymphocytes), red blood cell count $32 / \mu \mathrm{L}$, glucose $59 \mathrm{mg}$ per decaliter $(\mathrm{mg} / \mathrm{dL})$, protein $38 \mathrm{mg} / \mathrm{dL}$, negative meningitis/encephalitis panel (BioFire Diagnostics, Salt Lake City, UT) that includes VZV, and negative paraneoplastic panel (Mayo Clinic Laboratories). MRI brain with and without contrast demonstrated stable periventricular and subcortical white matter T2 hyperintensities. Continuous EEG was negative for seizures. She was treated with high dose IV steroids for 3 days and IV immunoglobulin (IVIG) for 5 days resulting in improvement of her mental status. However, 5 days after completing IVIG, she developed an increasing oxygen requirement, lethargy, and worsening aphasia. Chest X-ray revealed multifocal pneumonia, 
TABLE 2 | Neurological and non-neurological symptom onset and clinical presentation.

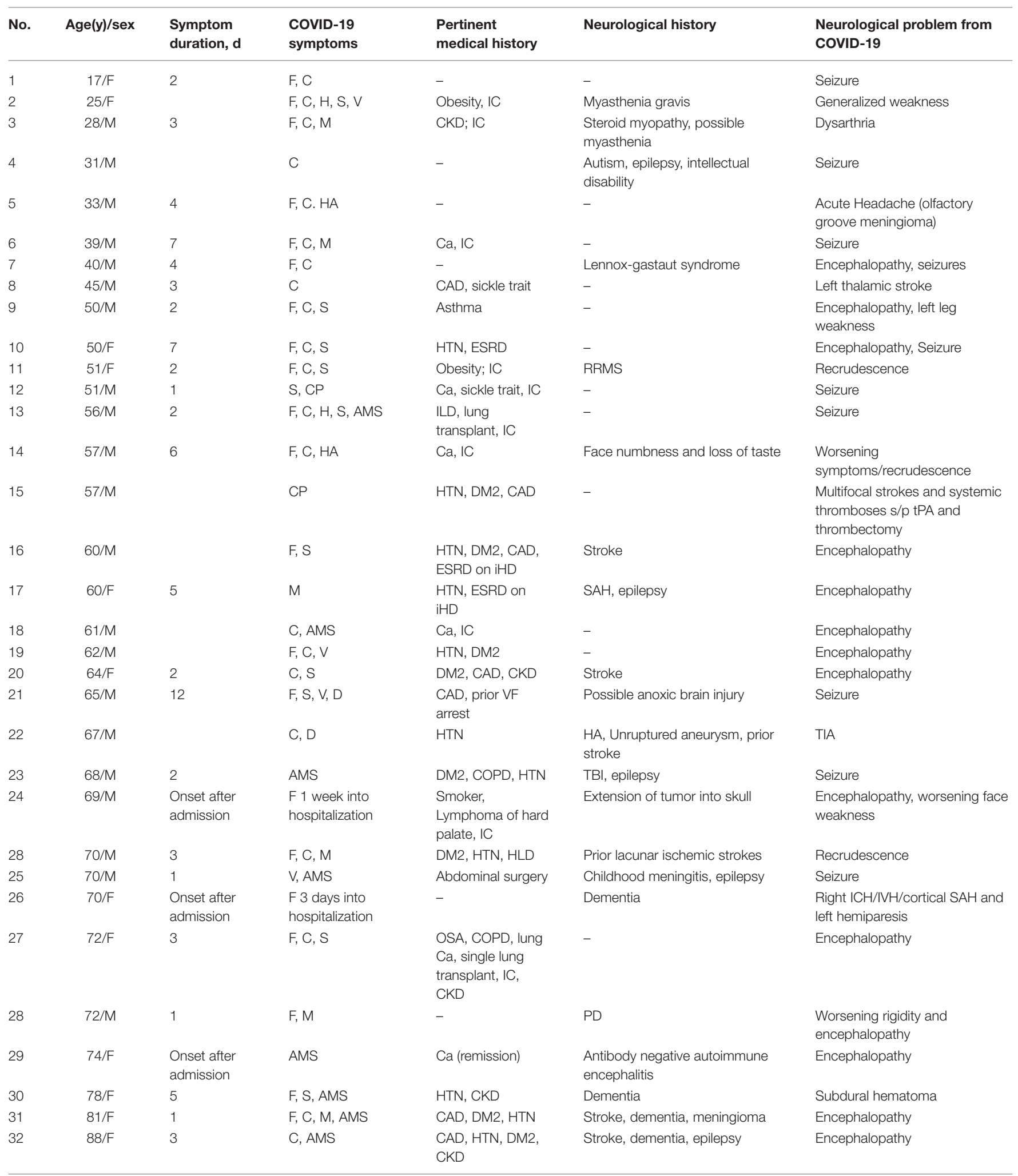

y, years; $d$, days; F, fever; $C$, cough; $T$, tachypnea; $H$, hypoxia; $S$, shortness of breath; $V$, vomiting; $M$, malaise; HA, headache; CP, chest pain; AMS, altered mental status; ID, intellectual disability; D, diarrhea; OSA, obstructive sleep apnea; COPD, chronic obstructive pulmonary disease; Ca, cancer; HTN, hypertension; DM, diabetes mellitus; ILD, interstitial lung disease; ESRD, end-stage renal disease; CKD, chronic kidney disease; iHD, intermittent hemodialysis; VF, ventricular fibrillation; RRMS, relapsing-remitting multiple sclerosis; ICH, intracerebral hemorrhage; IVH, intraventricular hemorrhage; SAH, subarachnoid hemorrhage; TIA, transient ischemic attack; IC, immunocompromised; TBI, traumatic brain injury; PD, Parkinson disease; tPA, tissue plasminogen activator; $s / p$, status post. 

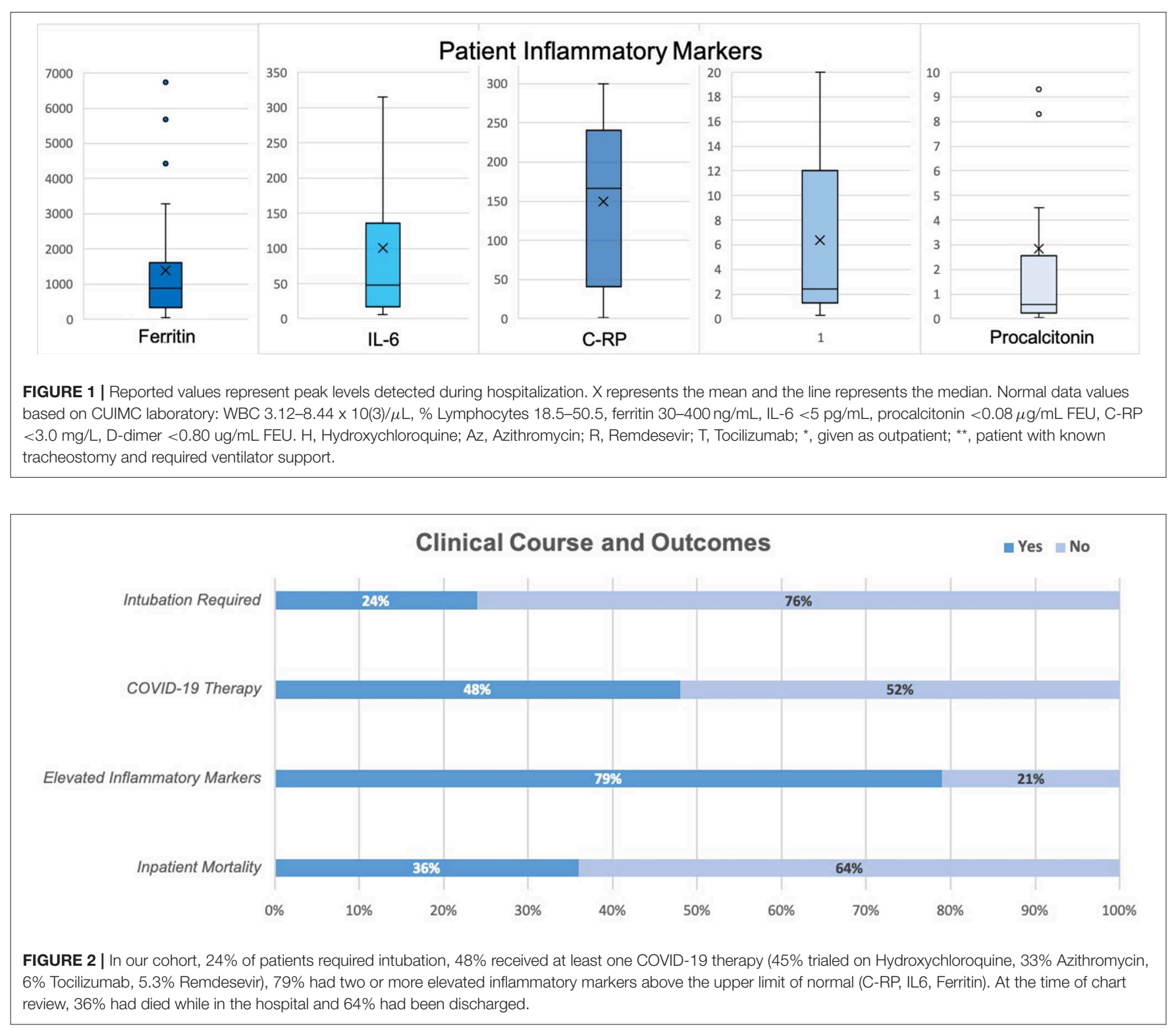

and nasopharyngeal swab was positive for SARS-CoV-2 on hospital day 17. Initial laboratory work-up was notable for new lymphopenia and elevated inflammatory markers. Her mental status decline was attributed to COVID-19 infection and recrudescence of her autoimmune encephalitis, so repeat LP was deferred. Azithromycin and hydroxychloroquine were started for SARS-Cov-2-directed experimental therapy. One day after initiation, she had significant improvement in her mental status and oxygenation. Nine days after symptom-onset, she returned to her neurological baseline.

\section{Case 15}

A 57-year-old man with history of hypertension, type 2 diabetes mellitus, and $\mathrm{CAD}$ was admitted with chest pain and found to have a myocardial infarction for which he underwent a 3-vessel coronary artery bypass grafting (CABG).
On post-operative day one (hospital day 6), a stroke code was called for acute onset right-sided weakness and obtundation. Following CT head, he was given tissue plasminogen activator (tPA). CT angiogram demonstrated vertebrobasilar occlusion. He underwent successful thrombectomy of the basilar artery. Angiogram at time of the thrombectomy demonstrated interval development of a new right internal carotid artery occlusion, which was also successfully treated. Follow-up CT head revealed a complete left posterior cerebral artery stroke with other smaller posterior circulation strokes with petechial hemorrhage in the right basal ganglia. Within $12 \mathrm{~h}$ of tPA administration, he developed thrombosis of the right brachial artery. He remained intubated. On hospital day 7, CT chest revealed bilateral consolidation concerning for pneumonia. $\mathrm{He}$ developed ARDS and tested positive for SARS-CoV2 on hospital day 11 . He sustained multi-organ failure 
due to mixed cardiogenic and septic shock and died on hospital day 25 .

\section{DISCUSSION}

The most common reasons for neurological evaluation in the setting of COVID-19 infection at our institution were encephalopathy, seizure, and stroke. Most of the patients in our case series developed neurological symptoms several days after COVID-19 symptom-onset and demonstrated elevated inflammatory markers. It is shown that patients with moderate to severe disease requiring hospitalization are more likely to develop a secondary, delayed hyperinflammatory state with elevated cytokine levels $(2,10)$. However, we are not able to comment on how these levels compare to COVID19 patients without neurologic symptoms in our inpatient population, COVID-19 patients in the outpatient setting, nor to healthy controls. This will be important to evaluate in future studies.

Severe SARS-CoV-2 infection can lead to impaired consciousness (4). Many factors can attribute to toxic-metabolic encephalopathy in patient with COVID-19, including hypoxia, organ dysfunction, and metabolic abnormalities (11). Many patients who require intubation and critical care also require prolonged sedation, immobilization, hospitalization, and social isolation, which also may contribute to toxic-metabolic encephalopathy (11). A postmortem brain examination has demonstrated brain edema and neuronal degeneration (12); however, these findings can be seen with hypoxia and metabolic abnormalities (13). Direct invasion of SARS-CoV-2 has not been demonstrated. The ability of our study to sample CSF in the initial stages of the pandemic at our institution were limited.

Acute symptomatic seizures can occur in the setting of systemic illness and sepsis (14), proposing a systemic inflammatory response leading to a decreased seizure threshold. The six patients who presented with first-time seizure of life had high inflammatory markers known to be elevated in COVID-19 infection, which suggests that these seizures could be categorized as provoked seizures in the setting of robust systemic inflammatory response. Other coronavirus infections have been associated with seizures (15). CSF was not obtained in our cases, so we could not exclude direct neuroinvasion or encephalitis as the cause of seizures.

Infection is a known trigger for stroke, potentially contributing up to one-third of ischemic strokes (16). Respiratory infections in particular have been associated with acute cerebrovascular events, most notably with influenzalike viral illnesses $(17,18)$. Stroke is emerging as a potential complication of infection with SARS-CoV-2. The incidence and relative risk of stroke from COVID-19 infection remains unknown. Retrospective studies in different countries have reported an incidence of $2.5-5.7 \%$ for acute stroke $(4,6,7)$. Case series in the United States have also demonstrated acute stroke occurring in young adults (9) and in patients with only mild respiratory symptoms from COVID-19 (8). These studies did not have comparative controls.
The mechanisms whereby COVID-19 may increase stroke risk include the following: Hypercoagulability (as demonstrated in part by elevated D-dimer) may contribute. Systemic inflammation and a cascade of pro-inflammatory cytokines (as demonstrated in part by elevated IL-6) may be a mediator between infection and hypercoagulability and may also destabilize atheromatous plaque $(4,19,20)$. Coronavirus-related cardiac injury may lead to embolic strokes (21). Direct viral invasion of the vascular wall via binding at and depletion of the angiotensin-converting enzyme 2 (ACE2) receptor may generate endothelial dysfunction, vasoconstriction, and stroke (22).

As in prior literature reviewing neurological manifestations of COVID-19 infection, our case series included instances of both ischemic stroke and intracranial hemorrhage, although our patients also had other cardiovascular risk factors for stroke. Due to our limited sample, we cannot comment on SARS-CoV-2 increasing the risk of acute stroke.

This study had several limitations. First, it was a retrospective study including patients at a single urban academic medical center. Only 33 cases were studied. While the goal of this study was to provide an account of our early experiences with the neurological manifestations of COVID-19 infection, these factors limit the generalizability of our data. This study included only cases for which neurology was consulted or involved in primary care of the patients, so these cases do not represent all neurological manifestations of COVID-19 infection. These have been reported elsewhere (4). Although in some cases diagnostic tests may have better elucidated mechanisms of neurologic symptoms in our patients, these were not uniformly performed due to either patient illness severity, PPE scarcity, or healthcare exposure. This was particularly true for lumbar punctures given the risk of prolonged exposure during the procedure. We therefore were unable to rule out alternative neurological infections as a cause of seizure, including in the three immunocompromised patients. Lastly, since this is a shortterm study period, long-term outcomes are not known.

Here we present the most common neurological problems during the first two weeks of the SARS-CoV-2 outbreak at our institution. COVID-19 infection did not change the overall management of neurological problems. All deaths were directly related to severe sepsis, ARDS, or cytokine storm causing refractory shock. Future studies will investigate if SARS$\mathrm{CoV}-2$ has direct neurological toxicity requiring alternative management strategies.

\section{DATA AVAILABILITY STATEMENT}

The raw data supporting the conclusions of this article will be made available by the authors, without undue reservation.

\section{ETHICS STATEMENT}

The studies involving human participants were reviewed and approved by Columbia University Irving Medical Center Institutional Review Board. Written informed consent to participate in this study was provided by the participants' legal 
guardian/next of kin. Written informed consent was obtained from the individual(s) for the publication of any potentially identifiable images or data included in this article.

\section{AUTHOR CONTRIBUTIONS}

SR, SE, HR, AM, and SS have full access to all of the data and takes full responsibility for the data presented, accuracy of the data analysis and interpretation, the conduct of the research design, and drafting of the manuscript for intellectual content. TW and $\mathrm{AB}$ has full access to all of the data and contributed to the conduct of the research design and statistical analysis. JD has full access to all of the data and takes full responsibility for the data presented and accuracy of the

\section{REFERENCES}

1. Borges do Nascimento IJ, Cacic N, Abdulazeem HM, von Groote TC, Jayarajah U, Weerasekara I, et al. Novel coronavirus infection (COVID-19) in humans: a scoping review and meta-analysis. J Clin Med. (2020) 9:E941. doi: 10.3390/jcm9040941

2. Huang C, Wang Y, Li X, Ren L, Zhao J, Hu Y, et al. Clinical features of patients infected with 2019 novel coronavirus in Wuhan, China. Lancet. (2020) 395:497-506. doi: 10.1016/S0140-6736(20)30183-5

3. Bohmwald K, Gálvez NMS, Ríos M, Kalergis AM. Neurologic alterations due to respiratory virus infections. Front Neurosci. (2018) 12:386. doi: $10.3389 /$ fncel.2018.00386

4. Mao L, Wang M, Chen S, He Q, Chang J, Hong C, et al. Neurological manifestations of hospitalized patients with COVID-19 in Wuhan, China: a retrospective case series study. JAMA Neurology. (2020) 77:1-9. doi: 10.1001/jamaneurol.2020.1127

5. Helms J, Kremer S, Merdji H, Clere-Jehl R, Schenck M, Kummerlen C, et al. Neurologic features in severe SARS-CoV-2 infection. N Engl J Med. (2020) 382:2268-70. doi: 10.1056/NEJMc2008597

6. Lodigiani C, Iapichino G, Carenzo L, Cecconi M, Ferrazzi P, Sebastian T, et al. Venous and arterial thromboembolic complications in COVID-19 patients admitted to an academic hospital in Milan, Italy. Thromb Res. (2020) 191:9-14. doi: 10.1016/j.thromres.2020.04.024

7. Klok FA, Kruip MJ, van der Meer NJ, Arbous MS, Gommers DAMPJ, Kant KM, et al. Incidence of thrombotic complications in critically ill ICU patients with COVID-19. Thromb Res. (2020) 191:145-7. doi: 10.1016/j.thromres.2020.04.013

8. Fara MG, Stein LK, Skliut M, Morgello S, Fifi JT, Dhamoon MS. Macrothrombosis and stroke in patients with mild Covid-19 infection. $J$ Thromb Haemost. (2020). doi:10.1111/jth.14938. [Epub ahead of print].

9. Oxley TJ, Mocco J, Majidi S, Kellner CP, Shoirah H, Singh IP, et al. Large-vessel stroke as a presenting feature of Covid-19 in the young. N Engl J Med. (2020) 382:e60. doi: 10.1056/NEJMc2009787

10. Ruan Q, Yang K, Wang W, Jiang L, Song J. Clinical predictors of mortality due to COVID-19 based on an analysis of data of 150 patients from Wuhan, China. Inten Care Med. (2020) 46:846-8. doi: 10.1007/s00134-020-05991-x

11. Kotfis K, Williams Roberson S, Wilson JE, Dabrowski W, Pun BT, Ely EW. COVID-19: ICU delirium management during SARS-CoV-2 pandemic. Crit Care. (2020) 24:176. doi: 10.1186/s13054-020-02882-x

12. Xu Z, Shi L, Wang Y, Zhang J, Huang L, Zhang C, et al. Pathological findings of COVID-19 associated with acute respiratory distress syndrome. Lancet Respir Med. (2020) 8:420-22. doi: 10.1016/S2213-2600(20)30076-X data analysis and interpretation. JB has full access to all of the data and takes full responsibility for the data presented, accuracy of the data analysis, interpretation, and revising the manuscript for intellectual content. JW and KT were responsible for conduct of the research design, analysis and interpretation of the data, and revising the manuscript for intellectual content. All authors contributed to the article and approved the submitted version.

\section{FUNDING}

This work was supported by National Institute of Health (NICHD/NIH 1R01HD074944-01 and NINDS/NIH K23 NS105935-01).

13. Stokum JA, Gerzanich V, Simard MJ. Molecular pathophysiology of cerebral edema. J Cereb Blood Flow Metab. (2016) 36:513-8. doi: 10.1177/0271678X15617172

14. Nwani PO, Nwosu MC, Nwosu MN. Epidemiology of acute symptomatic seizures among adult medical admissions. Epilepsy Res Treat. (2016) 2016:4718372. doi: $10.1155 / 2016 / 4718372$

15. Asadi-pooya AA. Seizures associated with coronavirus infections. Seizure. (2020) 79:49-52. doi: 10.1016/j.seizure.2020.05.005

16. Emsley HC, Hopkins SJ. Acute ischaemic stroke and infection: recent and emerging concepts. Lancet Neurol. (2008) 7:341-53. doi: 10.1016/S1474-4422(08)70061-9

17. Elkind MS. Why now? Moving from stroke risk factors to stroke triggers. Curr Opin Neurol. (2007) 20:51-7. doi: 10.1097/WCO.0b013e328012da75

18. Boehme AK, Luna J, Kulick ER, Kamel H, Elkind MSV. Influenza-like illness as a trigger for ischemic stroke. Ann Clin Transl Neurol. (2018) 5:456-63. doi: 10.1002/acn3.545

19. Zhou F, Yu T, Du R, Fan G, Liu Y, Liu Z, et al. Clinical course and risk factors for mortality of adult inpatients with COVID-19 in Wuhan, China: a retrospective cohort study. Lancet. (2020) 395:1054-62. doi: 10.1016/S0140-6736(20)30566-3

20. Mehta, P, McAuley, DF, Brown, M, Sanchez, E, Tattersall, RS, Manson, JJ. HLH across speciality collaboration UK. COVID-19: consider cytokine storm syndromes and immunosuppression. Lancet. (2020) 395:1033-4. doi: 10.1016/S0140-6736(20)30628-

21. Akhmerov, A, Marban, E. COVID-19 and the heart. Circ Res. (2020) 126:1443-55. doi: 10.1161/CIRCRESAHA.120.317055

22. Paniz-Mondolfi A, Bryce C, Grimes Z, Gordon RE, Reidy J, Lednicky J. Central nervous system involvement by severe acute respiratory syndrome coronavirus-2 (SARS-CoV-2). J Med Virol. (2020) 92:699-702. doi: $10.1002 /$ jmv.25915

Conflict of Interest: The authors declare that the research was conducted in the absence of any commercial or financial relationships that could be construed as a potential conflict of interest.

Copyright (C) 2020 Radmard, Epstein, Roeder, Michalak, Shapiro, Boehme, Wilson, Duran, Bain, Willey and Thakur. This is an open-access article distributed under the terms of the Creative Commons Attribution License (CC BY). The use, distribution or reproduction in other forums is permitted, provided the original author(s) and the copyright owner(s) are credited and that the original publication in this journal is cited, in accordance with accepted academic practice. No use, distribution or reproduction is permitted which does not comply with these terms. 